\title{
Single top quark production with CMS
}

\author{
Soureek Mitra* \\ (On behalf of CMS collaboration) \\ Tata Institute Of Fundamental Research \\ E-mail: soureek.mitra@cern.ch
}

\begin{abstract}
Measurements of single top quark production are presented, performed using CMS data collected in 2011, 2012 and 2015 at centre-of-mass energies of 7, 8 and $13 \mathrm{TeV}$ respectively. The cross sections for the electroweak production of single top quarks in the t-channel and in association with $\mathrm{W}$-boson are measured and the results are used to place constraints on the CKM matrix element $\mathrm{V}_{\mathrm{tb}}$. In the $\mathrm{t}$-channel the ratio of top and anti-top production cross sections is determined and compared with predictions from different parton density distribution functions. In the same channel, the inclusive cross-section in the fiducial volume is also measured. Measurements of top quark properties in single top quark production such as the top-quark polarisation, W-helicity in top quark decay and searches for anomalous couplings to photons are also presented. A search for the s-channel is also performed.
\end{abstract}

XXIV International Workshop on Deep-Inelastic Scattering and Related Subjects

11-15 April, 2016

DESY Hamburg, Germany

\footnotetext{
* Speaker.
} 


\section{Introduction}

The top quark is the heaviest among the elementary particles and has unique features in many ways. The top quark decays via electroweak interaction faster than typical hadronization time scale implying that it can exist as free quark before its decay. Due to this unique property, the top quark provides an excellent probe to signatures of physics beyond the Standard Model (SM) by comparing measurements with precise SM predictions. Top quarks are produced copiously in the proton-proton (pp) collision at the Large Hadron Collider (LHC). Single top quarks are produced in pp collisions through charged-current (CC) interaction via exchange of a virtual W-boson, unlike the dominant top pair $(\mathrm{t} \overline{\mathrm{t}})$ production process initiated by strong interaction. Single top production in pp collisions at LHC can be characterized via 3 modes: $\mathrm{t}$-channel, tW-channel and s-channel ordered according to decreasing cross-section which are shown in Figure 1.

Single top production is one of the important electroweak processes occurring at LHC. It provides a direct probe to the $\mathrm{tWb}$ coupling for any BSM contribution such as presence of flavor changing neutral current (FCNC), 4th generation, charged Higgs interaction etc. It allows measurement of fundamental parameters of standard model such as $\left|\mathrm{V}_{\mathrm{tb}}\right|$, top quark mass etc. Differential measurements in t-channel production mode can also provide powerful input to constrain b-quark parton distribution function (PDF) at high momentum fractions.

This paper discusses about the recent measurements performed by the Compact Muon Solenoid (CMS) [1] experiment at LHC of the cross-sections, both inclusive and differential of singlyproduced top quarks. The final section describes measurement of the properties of single top production which are sensitive to new physics model and a direct search for FCNC in an associated photon in addition to a top quark in the final state.

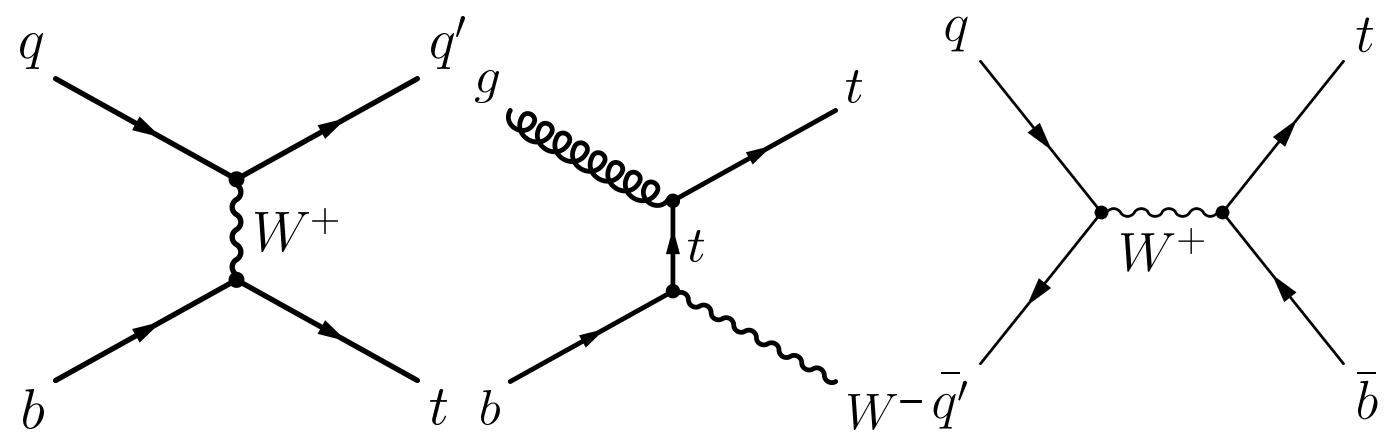

Figure 1: Feynman diagrams of electroweak single top production in standard model: t-channel (left), tW-channel (middle) and s-channel(right)

\section{Cross-section measurements}

The t-channel production mode is the most abundant among the 3 modes and therefore it is also the most studied. CMS has measured t-channel single top cross-section at 3 different centreof-mass (COM) energies, namely 7, 8 and $13 \mathrm{TeV}$. The measurement is based on the leptonic decay channel in which $\mathrm{W}$ boson produced from top decay, decays further to a muon $(\mu$ ) or electron (e) 
and one or more (via tau lepton cascade decay chains, e.g., $\mathrm{t} \rightarrow \tau \rightarrow \mu$ ) associated neutrino. The experimental signature of t-channel is characterized by the presence of exactly one energetic isolated lepton ( $\mu$ or e), two energetic jets and large missing transverse energy $\left(\mathrm{E}_{T}^{\text {Miss }}\right)$ or transverse mass $\left(\mathrm{m}_{T}^{W}\right)$ of lepton and $\mathrm{E}_{T}^{\text {Miss }}$ system. One of the jets is required to pass a tight threshold of a neural network $(\mathrm{NN})$ based b-tagging discriminator and it is interpreted as coming from top quark decay and the other (failing the same threshold) as originating from the spectator quark recoiling against top in high pseudo-rapidity region. $\mathrm{t} \bar{t}, \mathrm{~W}$ boson produced in association with jets (W+Jets) are the most dominant background to this final state. Orthogonal control regions with different jet and b-tagged jet multiplicity are used to estimate these backgrounds or validate Monte Carlo (MC) models used for their predictions. QCD multijet events also constitute a non-negligible background and a reliable estimation of this background is performed using a control region by inverting lepton isolation requirement while fulfilling all other selection criteria. The signal cross-section is extracted by profile likelihood fit to a multivariate discriminator $(13 \mathrm{TeV})$ or spectator jet $|\eta|$ (8 $\mathrm{TeV}$ ) [2] [3]. An estimate of $\left|\mathrm{V}_{\mathrm{tb}}\right| \mathrm{CKM}$ matrix element at both COM energies is available by comparing measurements with theory predictions. CMS has also measured the ratio $\left(\mathrm{R}_{\mathrm{t}-\mathrm{ch}}=\frac{\sigma_{t}}{\sigma_{\mathrm{t}}}\right)$ of production rate of top and anti-top in t-channel with 8 and $13 \mathrm{TeV}$ data. The measured values agree within uncertainties in both COM energy and already disfavoring some of the PDF sets. A differential cross-section measurement of t-channel production with top-quark $\mathrm{p}_{T}^{t o p}$ and rapidity $\left(\left|\mathrm{y}_{t o p}\right|\right)$ has been performed in CMS at $\sqrt{s}=8 \mathrm{TeV}$ [4]. In this analysis, unfolding is performed in a signal enriched sample obtained by cutting on NN based discriminator (NN output $>0.3(0.4)$ for $\mu$ (e) in final state). Measurements have overall good agreement with different $\mathrm{MC}$ prediction, though aMC@NLO [5] in 4 flavour scheme [6] with PYTHIA8 [7] showering is observed to have better agreement with measurement in the tail region of top $\mathrm{p}_{T}$. Fiducial cross-section measurement in $\mathrm{t}-$ channel to compare with theoretical predictions is performed with the data collected at $\sqrt{s}=8 \mathrm{TeV}$ [8]. For this, a fiducial volume is defined with selection cuts on generator level particles similar to the reconstructed objects and the measurement is restricted to this well-defined fiducial volume. The aim of this analysis is to reduce the dependency of the final result on the acceptance-based extrapolation and test different MC predictions. Signal modeling, jet energy scale (JES), PDF and renormalization/factorization scale uncertainties in signal and backgrounds ( $\mathrm{t} \overline{\mathrm{t}}$ and $\mathrm{W}+\mathrm{Jets}$ ) have dominant contribution to the total uncertainty of the measurements of inclusive and differential cross-sections in t-channel.

CMS has reported first observation of tW-channel with data collected at $\sqrt{s}=8 \mathrm{TeV}$ [9]. It is the second most abundant single top production mode at LHC. The analysis looks into events having two charged leptons (ee, e $\mu$ and $\mu \mu$ ) in final states. Each of the 3 possible di-lepton final states is further categorized in bins of jet multiplicity and b-tagged jet multiplicity and the sample with two charged leptons, only one jet which is b-tagged has been identified as signal-enriched sample. $t \bar{t}$ constitutes the dominant background to this process and control regions with different jet and b-jet multiplicities has been used to estimate this background. A boosted decision tree (BDT) based discriminator has been used in likelihood fit to extract signal cross-section which has significance of 6.1 standard deviations. Uncertainties due to matrix element - parton shower (ME-PS) matching threshold, renormalization/factorization scale, top quark mass, fit statistics etc. contribute significantly to the total uncertainty.

Single top quark production in s-channel is initiated, at leading order (LO), by q $\bar{q}^{\prime}$ annihilation as 
shown in Figure 1 and therefore is less abundant in pp collisions than $p \bar{p}$ collisions at all energies and the cross-section scales less with increasing COM energy when compared to other single top channels and tt. Its observation is very recently reported by the combination of Tevatron measurements [10]. A final state consisting of one charged lepton (e or $\mu$ ), two b-tagged jets and large $\mathrm{E}_{T}^{\text {Miss }}$ or $\mathrm{m}_{T}^{W}$ is searched for s-channel single top signal in CMS with 7 and $8 \mathrm{TeV}$ data. $\mathrm{t} \overline{\mathrm{t}}, \mathrm{W}+\mathrm{Jets}$, t-channel single top and QCD multijet are the dominant backgrounds to this final state. BDT discriminators are trained in signal region as well as in control regions with different jet and b-tagged jet multiplicities to control the backgrounds. Simultaneous likelihood fits to BDT discriminators in signal and control regions is performed to extract s-channel cross-section. The signal significance is 2.5 standard deviations, and the upper limit on the rate relative to SM expectation is set to be 4.7 with 95\% confidence level with a combination of measurements performed at 7 and $8 \mathrm{TeV}$ [11]. All inclusive cross-section measurements in CMS are summarized in Figure 2.

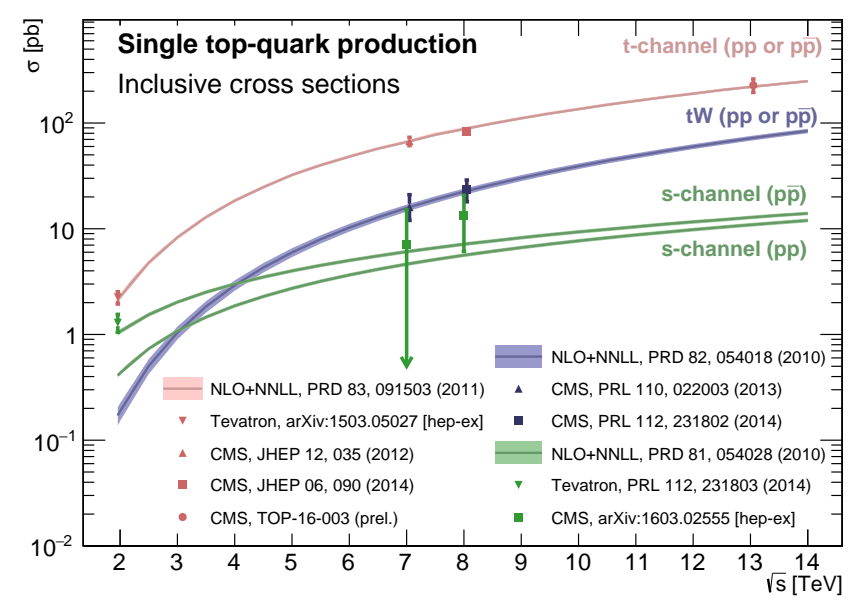

Figure 2: Summary of single top cross section measurements by CMS, as function of centre-ofmass energy

\section{Properties measurement}

The top quark provides a unique opportunity to study "bare" quark properties due to the neat separation of the relevant time scales: the production time $\left(\approx \frac{1}{m_{\text {top }}}\right.$, where $\left.\mathrm{m}_{\text {top }} \approx 175 \mathrm{GeV}\right)$ is two orders of magnitude smaller than its lifetime $\left(\frac{1}{\Gamma_{\text {top }}}\right.$, where $\left.\Gamma_{\text {top }} \approx 2 \mathrm{GeV}\right)$, which is an order of magnitude smaller than the hadronization time-scale $\left(\approx \frac{1}{\Lambda_{Q C D}}\right.$ with $\left.\Lambda_{Q C D} \approx 0.2 \mathrm{GeV}\right)$, which in turn, is also an order of magnitude smaller than the spin decorrelation time $\left(\approx \frac{m_{t o p}}{\Lambda_{O C D}^{2}}\right)$. This implies that top decay products remember its helicity information since its lifetime is shorter than the spin decorrelation time scale. This provides additional powerful tool to search for new physics in single top-quark production via the $\mathrm{t}$ channel. The $\mathrm{V}$-A coupling structure of $\mathrm{tWb}$ vertex in SM predicts that the top quark is polarised fully in the momentum direction of the quark recoiling against top [12] [13]. New physics can show up if depolarisation is observed in the electroweak 
production or decay of the top quark which indicate an alteration of the tWb coupling. In top rest frame, angular distribution $\left(\theta_{X}^{*}\right)$ of decay product $X$ with respect to spin direction of top-quark can be written as

$$
\frac{1}{\sigma} \frac{d \sigma}{d \cos \theta_{X}^{*}}=\frac{1}{2}\left(1+P_{t}^{(S)} \alpha_{X} \cos \theta_{X}^{*}\right)=\frac{1}{2}+A_{X} \cos \theta_{X}^{*}
$$

where $\mathrm{A}_{X}=\frac{1}{2} P_{t}^{(S)} \alpha_{X}$ with $\mathrm{P}_{t}^{(S)}$ being the top polarisation (due to production vertex) along its spin direction, $\alpha_{X}$ being the spin-analysing power of the decay particle $\mathrm{X}(\approx 100 \%$ for charged lepton $)$ and $\theta_{X}^{*}$ being the angle between decay product $X$ and the recoiling jet in the top-quark rest frame. Both $\mathrm{P}_{t}^{(S)}$ and $\alpha_{X}$ can be affected due to change in coupling structure in the tWb vertex. The SM expectation is $\mathrm{P}_{t}^{(S)} \alpha_{X} \approx 1$ for muon decay product $(\mathrm{X}=\mu)$. A dedicated measurement of singletop polarisation has been performed by CMS with $8 \mathrm{TeV}$ data with a model-independent selection targeting t-channel single top events [14]. The observed distribution of $\cos \theta_{\mu}^{*}$ is used infer the differential cross-section as a function of $\cos \theta_{\mu}^{*}$ which is found to have linear behavior as expected in SM but with a slope $\mathrm{A}_{\mu}=0.26 \pm 0.11$ which is 2 standard deviations away form SM prediction $\left(\mathrm{A}_{\mu}^{S M}=0.44\right)$.

The W-boson helicity fractions in top-quark decay are sensitive to the non-SM behaviour of the tWb vertex. CMS has, for the first time, performed a measurement of the W-boson helicity fractions in a phase-space region defined by a selection enriched in t-channel single top-quark events in the lepton + jets final state, statistically independent from the measurements performed in tit optimized selection, with a precision comparable to that of the measurements from $t \bar{t}$ [15]. The functional form of the top quark partial decay width can be written as a function of the right-handed $\left(\mathrm{F}_{R}\right)$, left-handed $\left(\mathrm{F}_{L}\right)$ and longitudinal $\left(\mathrm{F}_{0}\right)$ helicity fractions of the W-boson as

$$
\frac{1}{\Gamma} \frac{d \Gamma}{d \cos \theta_{\ell}^{*}}=\frac{3}{8}\left(1-\cos \theta_{\ell}^{*}\right)^{2} F_{L}+\frac{3}{4} \sin ^{2} \theta_{\ell}^{*} F_{0}+\frac{3}{8}\left(1+\cos \theta_{\ell}^{*}\right)^{2} F_{R}
$$

The helicity angle $\theta_{\ell}^{*}$ is defined as the angle between W-boson momentum in top-quark rest frame and momentum of down-type fermion (lepton in this case) in $\mathrm{W}$ rest frame. The measurement is based on extracting the helicity fractions $\mathrm{F}_{L}$ and $\mathrm{F}_{0}$ from a maximum likelihood fit to the data distribution of $\cos \theta_{\ell}^{*}$ and estimate $\mathrm{F}_{R}$ from the condition $\Sigma F_{i}=1 . \mathrm{F}_{L}, \mathrm{~F}_{0}$ and W+Jets normalization are kept free to float during maximum likelihood fit to data. The results are found to be in good agreement with SM predictions accurate upto next-to-next-leading-order (NNLO) calculation. The helicity measurements are also interpreted to set limits on the real part of the anomalous tensor-like couplings.

A unique search for FCNC through single top quark production in association with a photon has been performed with $8 \mathrm{TeV}$ data [16]. The search for $\mathrm{t} \gamma$ events where $\mathrm{t} \rightarrow \mathrm{Wb}$ and $\mathrm{W} \rightarrow \mu \nu$ is conducted in final states with an isolated muon, a high $\mathrm{p}_{T}$ photon, at least one hadronic jet with at most one b-tagged jet and $\mathrm{E}_{T}^{\text {Miss }}$ as shown in Figure 3. W+ $\gamma+$ Jets and $\mathrm{W}+$ Jets constitute the most dominant backgrounds and they are estimated from data. BDT based discriminator trained to separate FCNC signal from SM backgrounds is fitted to data. No evidence of single top quark production 
in association with a photon through a FCNC is observed. Upper limits at 95\% confidence level are set on the tu $\gamma$ and tc $\gamma$ anomalous couplings and translated into upper limits on the branching fraction of the FCNC top quark decays: $\mathrm{B}(\mathrm{t} \rightarrow \mathrm{u} \gamma)<1.3 \times 10^{-4}$ and $\mathrm{B}(\mathrm{t} \rightarrow \mathrm{c} \gamma)<1.7 \times 10^{-3}$. These are the most stringent limit currently available.
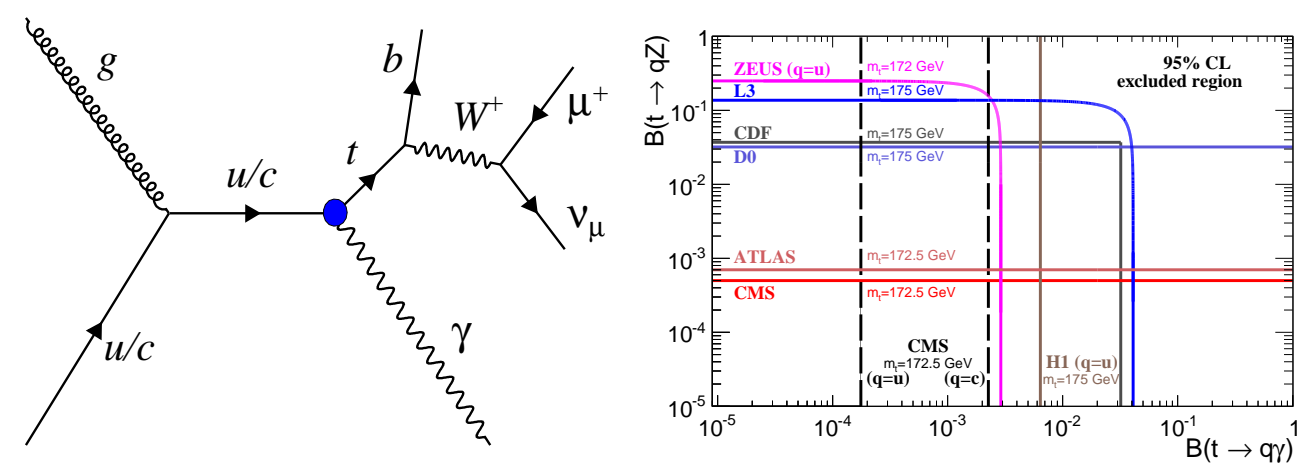

Figure 3: Diagram for FCNC search in single top quark production in association with a photon (left) and summary of FCNC measurements in the $\mathrm{BR}(\mathrm{t} \rightarrow \mathrm{Zq})$ vs $\mathrm{BR}(\mathrm{t} \rightarrow \gamma \mathrm{q})$ plane (right)

\section{Conclusion}

$\mathrm{t}$-channel single top production has already entered the precision regime within few years of its experimental observation [17] [18]. Current measurements are mostly dominated by uncertainties of theoretical origin such as signal modeling, renormalization scale, PDF etc. Better understanding of $\mathrm{MC}$ generators and more accurate predictions are required to further improve the precision of measurements. CMS is actively participating in LHC combination of single top cross-sections with Run1 data. tW-channel is soon expected to be included in the precision regime with $13 \mathrm{TeV}$ data due to increased cross-section. Observation of single top production in s-channel has eluded CMS at 7 and $8 \mathrm{TeV}$ and it continues to be a difficult hurdle to overcome at $\sqrt{s}=13 \mathrm{TeV}$. Improvement on the limits on anomalous coupling is expected with more data. The whole community is eagerly waiting for exciting surprises that nature has in store during Run2 of LHC at $13 \mathrm{TeV}$.

\section{References}

[1] CMS collaboration, S. Chatrchyan et al., The CMS experiment at the CERN LHC, JINST 3 (2008) S08004.

[2] CMS collaboration, V. Khachatryan et al., Measurement of the t-channel single-top-quark production cross section and of the $\left|V_{t b}\right|$ CKM matrix element in pp collisions at $\sqrt{s}=8$ TeV, JHEP 06 (2014) 090, [1403. 7366].

[3] CMS collaboration, Measurement of the inclusive cross section of single top quark production in the $t$ channel at $\sqrt{s}=13 \mathrm{TeV}$, Tech. Rep. CMS-PAS-TOP-16-003, CERN, Geneva, 2016. 
[4] CMS collaboration, Single top t-channel differential cross section at $8 \mathrm{TeV}$, Tech. Rep. CMS-PAS-TOP-14-004, CERN, Geneva, 2014.

[5] J. Alwall, R. Frederix, S. Frixione, V. Hirschi, F. Maltoni, O. Mattelaer et al., The automated computation of tree-level and next-to-leading order differential cross sections, and their matching to parton shower simulations, JHEP 07 (2014) 079, [1405.0301].

[6] F. Maltoni, G. Ridolfi and M. Ubiali, b-initiated processes at the LHC: a reappraisal, JHEP 07 (2012) 022, [1203. 6393].

[7] T. Sjostrand, S. Mrenna and P. Z. Skands, A Brief Introduction to PYTHIA 8.1, Comput. Phys. Commun. 178 (2008) 852-867, [0 710 . 3820].

[8] CMS collaboration, Fiducial t channel single top-quark cross section at $8 \mathrm{TeV}$, Tech. Rep. CMS-PAS-TOP-15-007, CERN, Geneva, 2015.

[9] CMS collaboration, S. Chatrchyan et al., Observation of the associated production of a single top quark and $a \mathrm{~W}$ boson in pp collisions at $\sqrt{s}=8 \mathrm{TeV}$, Phys. Rev. Lett. 112 (2014) 231802, [1401.2942].

[10] CDF, D0 collaboration, T. A. Aaltonen et al., Observation of s-channel production of single top quarks at the Tevatron, Phys. Rev. Lett. 112 (2014) 231803, [1402. 5126].

[11] CMS collaboration, V. Khachatryan et al., Search for s channel single top quark production in pp collisions at $\sqrt{s}=7$ and $8 \mathrm{TeV}, 1603.02555$.

[12] G. Mahlon and S. J. Parke, Single top quark production at the LHC: Understanding spin, Phys. Lett. B476 (2000) 323-330, [hep-ph/ 9912458 ].

[13] M. Jezabek and J. H. Kuhn, V-A tests through leptons from polarized top quarks, Phys. Lett. B329 (1994) 317-324, [hep-ph/9403366].

[14] CMS collaboration, V. Khachatryan et al., Measurement of top quark polarisation in t-channel single top quark production, JHEP 04 (2016) 073, [1511.02138].

[15] CMS collaboration, V. Khachatryan et al., Measurement of the $W$ boson helicity in events with a single reconstructed top quark in pp collisions at $\sqrt{s}=8 \mathrm{TeV}$, JHEP 01 (2015) 053, [1410.1154].

[16] CMS collaboration, V. Khachatryan et al., Search for anomalous single top quark production in association with a photon in pp collisions at $\sqrt{s}=8 \mathrm{TeV}$, JHEP 04 (2016) 035, [1511.03951].

[17] D0 collaboration, V. M. Abazov et al., Observation of Single Top Quark Production, Phys. Rev. Lett. 103 (2009) 092001, [0 903.085 0].

[18] CDF collaboration, T. Aaltonen et al., First Observation of Electroweak Single Top Quark Production, Phys. Rev. Lett. 103 (2009) 092002, [0 903.088 5]. 